\title{
Towards a better classification of unclear eruptive variables: the cases of V2492 Cyg, V350 Cep, and ASASSN-15qi
}

\author{
R. Jurdana-Šepić ${ }^{1}$, U. Munari ${ }^{2}$, S. Antoniucci ${ }^{3}$, T. Giannini ${ }^{3}$, and D. Lorenzetti ${ }^{3}$ \\ ${ }^{1}$ Physics Department, University of Rijeka, Radmile Matejčić, 51000 Rijeka, Croatia \\ e-mail: jurdana@phy.uniri.hr \\ 2 INAF - Osservatorio Astronomico di Padova, Via dell'Osservatorio 8, Asiago (VI) 36012, Italy \\ ${ }^{3}$ INAF - Osservatorio Astronomico di Roma, Via Frascati 33, Monte Porzio Catone 00078, Italy
}

Received 19 October 2017 / Accepted 18 January 2018

\begin{abstract}
Context. Eruptive variables are young stars that show episodic variations of brightness: EXors/FUors variations are commonly associated with enhanced accretion outbursts occurring at intermittent cadence of months/years (EXors) and decades/centuries (FUors). Variations that can be ascribed to a variable extinction along their line of sight are instead classified as UXors.

Aims. We aim at investigating the long-term photometric behaviour of three sources classified as eruptive variables. We present data from the archival plates of the Asiago Observatory relative to the fields where the targets are located. For the sake of completeness we have also analysed the Harvard plates of the same regions that cover a much longer historical period, albeit at a lower sensitivity, however we are only able to provide upper limits.

Methods. A total of 273 Asiago plates were investigated, providing a total of more than 200 magnitudes for the three stars, which cover a period of about $34 \mathrm{yr}$ between 1958 and 1991. We have compared our data with more recently collected literature data.

Results. Our plates analysis of V2492 Cyg provides historical upper limits that seem not to be compatible with the level of the activity monitored during the last decade. Therefore, recently observed accretion phenomena could be associated with the outbursting episodes, more than repetitive obscuration. While a pure extinction does not seem the only mechanism responsible for the ASASSN-15qi fluctuations, it can account quite reasonably for the recent V350 Cep variations.
\end{abstract}

Key words. stars: pre-main sequence - stars: variables: T Tauri, Herbig Ae/Be - catalogs - stars: individual: V2492 Cyg stars: individual: V350 Cep - stars: individual: ASASSN-15qi

\section{Introduction}

The last phases of the matter accretion onto young stellar objects (YSOs) occur through intermittent bursts of the mass-accretion rate that consequently provoke a remarkable increase of brightness, mainly at optical and near-infrared (NIR) frequencies. A detailed description of the current knowledge of the accretion process onto young stars has recently been given by Hartmann et al. (2016). According to a general consensus these eruptive events are classified into two major classes, FUors and EXors, which, at the moment, globally amount to only a few tens of objects (see also the review by Audard et al. 2014). FUors (Hartmann \& Kenyon 1985) are characterized by bursts of long duration (tens of years) with accretion rates of the order of $10^{-4}-10^{-5} M_{\odot} \mathrm{yr}^{-1}$ and spectra dominated by absorption lines. EXors (Herbig 1989; Lorenzetti et al. 2012) show shorter outbursts (between months and one year) with a recurrence time of years, have accretion rates of the order of $10^{-6}-10^{-7} M_{\odot} \mathrm{yr}^{-1}$, and are characterized by emission line spectra (e.g. Herbig 2008; Lorenzetti et al. 2009; Kóspál et al. 2011; Sicilia-Aguilar et al. 2012; Antoniucci et al. 2013, 2014; Giannini et al. 2016). Bursts of both classes are thought to be triggered by instabilities originating in the disk itself (Zhu et al. 2009; D'Angelo \& Spruit 2010, 2012), in particular in the inner connection region between star and disk. To have a more complete account for the scenario of the young variables, UXors objects must also be considered (Grinin 1988), whose brightness variations are related to orbiting dust structures that move along the line of sight.

Observational activity is often oriented to obtain a quick classification by considering the mentioned properties as overstrict, whereas it is becoming increasingly clear that a large variety of different cases exists, for which it is difficult to discriminate between the three classes (see e.g. the case of V1647 Ori, whose variability is loosely defined Aspin 2011; Kóspál et al. 2011; and, more recently, that of V346 Nor - Kraus et al. 2016; Kóspál et al. 2017). Sometimes even the origin of the observed variability is a matter of debate (see e.g. the case of GM Cep - Sicilia-Aguilar et al. 2008; Xiao et al. 2010). These dubious circumstances arise from observations that often rely on a single (or a few) event(s) and not on a long-lasting photometric and/or spectroscopic monitoring. Remarkably, a single outburst does not allow us to ascertain if the object will remain or not in its higher state; analogously, from a single fading, we cannot determine whether we are looking at a typically bright object subject to repetitive obscuration or, conversely, at a quiescent object that undergoes recurrent outbursts.

One method to ameliorate the classification process involves investigating (whenever it is possible) the past history of the objects. A long-lasting (up to half century or more) monitoring can be obtained by digging into the plate archives, which allow us to build up light curves in the optical bands, where the eruptive variables present their largest fluctuations. This method has 
proven to be very efficient for studying the eruptive variables, as recently demonstrated for the FUor V960 Mon (Jurdana-Šepić \& Munari 2016) and for the EXors GM Cep (Xiao et al. 2010) and V1118 Ori (Jurdana-Šepić et al. 2017).

In the following, we examine deep BVRI plates taken between 1958 and 1993 with the Asiago Schmidt telescopes, of three young variables classified as eruptive objects, namely V2492 Cyg, V350 Cep, and ASASSN-15qi, which for different reasons deserve a more in-depth analysis in order to ascertain their nature. We aim at improving the characterization of their quiescent phase, which will represent a useful reference for studies analysing future outbursts and the physical changes induced by these events.

To expand our search to a more distant past (up to $~ 1890$ ), we have also examined all the deepest plates covering our three program stars that we have been able to locate in the plate stack of the Harvard College Observatory (HCO). None of them, unfortunately, turned out to be as deep as the Asiago plates, their limiting recorded brightness being typically several magnitudes brighter.

The paper is organised as follows: our sample is presented in Sect. 2; the adopted method and the obtained BVRI photometry are presented in Sects. 3 and 4. Section 5 gives the analysis and discussion of the obtained results, while our concluding remarks are given in Sect. 6.

\section{The investigated sample}

\subsection{V2492 Cyg}

The object V2492 Cyg $\left(\alpha_{2000}=20^{\mathrm{h}} 51^{\mathrm{m}} 26.23^{\mathrm{s}}, \quad \delta_{2000}\right.$ $\left.+44^{\circ} 05^{\prime} 23.9^{\prime \prime}\right)$ is located in the North American-Pelican Nebula at an estimated distance of 550 pc (Bally \& Reipurth 2003). Its first outburst on 2010 August 23 was discovered by Itagaki \& Yamaoka (2010) and the brightness increase of 5 mag lasted about 10 months. Covey et al. (2011) independently discovered the V2492 Cyg outburst: their optical and near-IR spectroscopy revealed a rich emission-line spectrum that allowed them to derive a mass-accretion rate of $2.5 \times 10^{-7} M_{\odot} \mathrm{yr}^{-1}$. Blue-shifted absorption at several hundred $\mathrm{km} \mathrm{s}^{-1}$ and the presence of typical tracers of shocked gas (such as $\mathrm{H}_{2}$, [SII] and [FeII]) both indicate a substantial amount of outflowing matter. The same spectroscopic evidence was confirmed by Aspin (2011), who also considered archival data (2009-2010) to investigate the pre-outburst nature of V2492 Cyg. His conclusions favour an EXor classification for V2492 Cyg, although the source appears to be significantly younger than other members typical of the class. After its fading, the source underwent additional episodes of brightening followed by dimming events; all these phases were extensively sampled by Hillenbrand et al. (2013), who detected neutral and singly ionized atomic species likely formed in an accretion flow, but also identified a behaviour attributable to rotating circumstellar disk material that causes the semi-periodic dimming. Therefore they concluded that V2492 Cyg simultaneously displays accretion-driven and extinction-driven fluctuations. Kóspál et al. (2013), observing the source between $0.55 \mu \mathrm{m}$ and $160 \mu \mathrm{m}$, regard V2492 Cyg as an UXor-type system. The most recent (Nov. 2016-Mar. 2017) peak of brightness, at a level never reached before, has been announced by Ibryanov \& Semkov (2017) and spectroscopically studied at high resolution by Munari et al. (2017) and Giannini et al. (2018), who confirm the great deal of emission lines as presented by Aspin (2011), but with absorptions that are now far stronger.

\subsection{V350 Cep}

Herbig (2008) provides a summary of the past history of the late-type (M2) pre-main sequence star V350 Cep $\left(\alpha_{2000}=\right.$ $\left.21^{\mathrm{h}} 43^{\mathrm{m}} 00.00^{\mathrm{s}}, \delta_{2000}+66^{\circ} 11^{\prime} 28.0^{\prime \prime}\right)$ in NGC 7129 , a region of active star formation (Dahm \& Hillenbrand 2015 and references therein) located at $1150 \mathrm{pc}$ (Straižys et al. 2014). The target was undetected (i.e. $B>21 \mathrm{mag}$ ) on the 1954 Palomar plates, but a brightness (about $B=17.5$ ) was found in the middle of the 1970s. From 1978 until 2004, V350 Cep remained at the same level of brightness $(B \sim 17)$ as shown by Pogosyants \& Yu (1991), and Semkov (2004, and references therein). As a consequence, all the following spectroscopic investigations were obtained at a high level of brightness, and, substantially, all confirm the classical T Tauri (CTTS) nature of V350 Cep. Herbig (2008) also examined the high-resolution spectrum discussing important dynamical details, suggesting that V350 cannot be classified as an EXor source. Ibryanov et al. (2014) provide the result of their $U B V R I$ photometry in the period 2004-2014, during which the star maintained its maximum brightness (for a complete view of the historical light curve, see their Fig. 1). The $V$-band light curve retrievable from the ASASSN $^{1}$ database confirms that V350 Cep is even now at the same high level of brightness. The simultaneous presence of an emission line spectrum and the apparent lack of a repetitive activity represent contrasting evidences toward a certain classification of V350 Cep. Further ambiguities stem from the recent observations by Semkov et al. (2017), who registered a deep fading $(\Delta B=2.16, \Delta V=1.77)$ in the period between March and May 2016 , followed by a quick restoration (occurring in the second half of 2016) of its maximum brightness. They suggest that such an event is compatible with an obscuration from clumps orbiting the star or with a decrease in the accretion rate, or with a combination of both mechanisms.

\subsection{ASASSN-15qi}

ASASSN-15qi $\left(\alpha_{2000}=20^{\mathrm{h}} 56^{\mathrm{m}} 08.82^{\mathrm{s}}, \delta_{2000}+51^{\circ} 31^{\prime} 04.1^{\prime \prime}\right)$ recently (2015 Oct $2 \mathrm{nd} / 3 \mathrm{rd}$ ) underwent a sudden brightening $(\Delta V=3.5 \mathrm{mag})$ in less than one day. Strong P-Cyg profiles with red emission wings traced a very fast wind with a velocity up to $1000 \mathrm{~km} \mathrm{~s}^{-1}$ that faded while the central source returned to quiescence (Maehara et al. 2015; Herczeg et al. 2016). The distance estimate for ASASSN-15qi is around $3.24 \mathrm{kpc}$. Assuming the values of the pre-outburst photometry, Hillenbrand et al. (2015) derived an $\mathrm{A}_{V}$ of about $5 \mathrm{mag}$ and a luminosity of $\sim 125 \mathrm{~L}_{\odot}$. Consequently, they also derived a progenitor source roughly 2.5-3.0 $M_{\odot}$, and concluded the source cannot be easily classified into the FUor and EXors classes according to our current knowledge of the defining criteria. Connelley et al. (2015) obtained an early near-IR spectrum showing a strong veiling, a number of emission lines (with strong P-Cyg), and $\mathrm{CO}$ bandheads in emission. They also revealed a very faint nebulosity seen only in the $J$ band), likely due to a reflection nebula. A thorough collection of UV, optical, near- and mid-IR and sub-mm observations, together with archival photometry (mainly obtained in 2000 and 2015), was presented by Herczeg et al. (2016). They were able to build-up reliable Spectral Energy Distributions (SEDs) of ASASSN-15qi during quiescence, outburst, peak, decay, and quiescence again. They also provide an accurate description of the dynamical events during the different phases. Nevertheless, the observations cannot easily be explained in

\footnotetext{
1 All-Sky Automated Survey for Supernovae

(https://asas-sn.osu.edu/)
} 


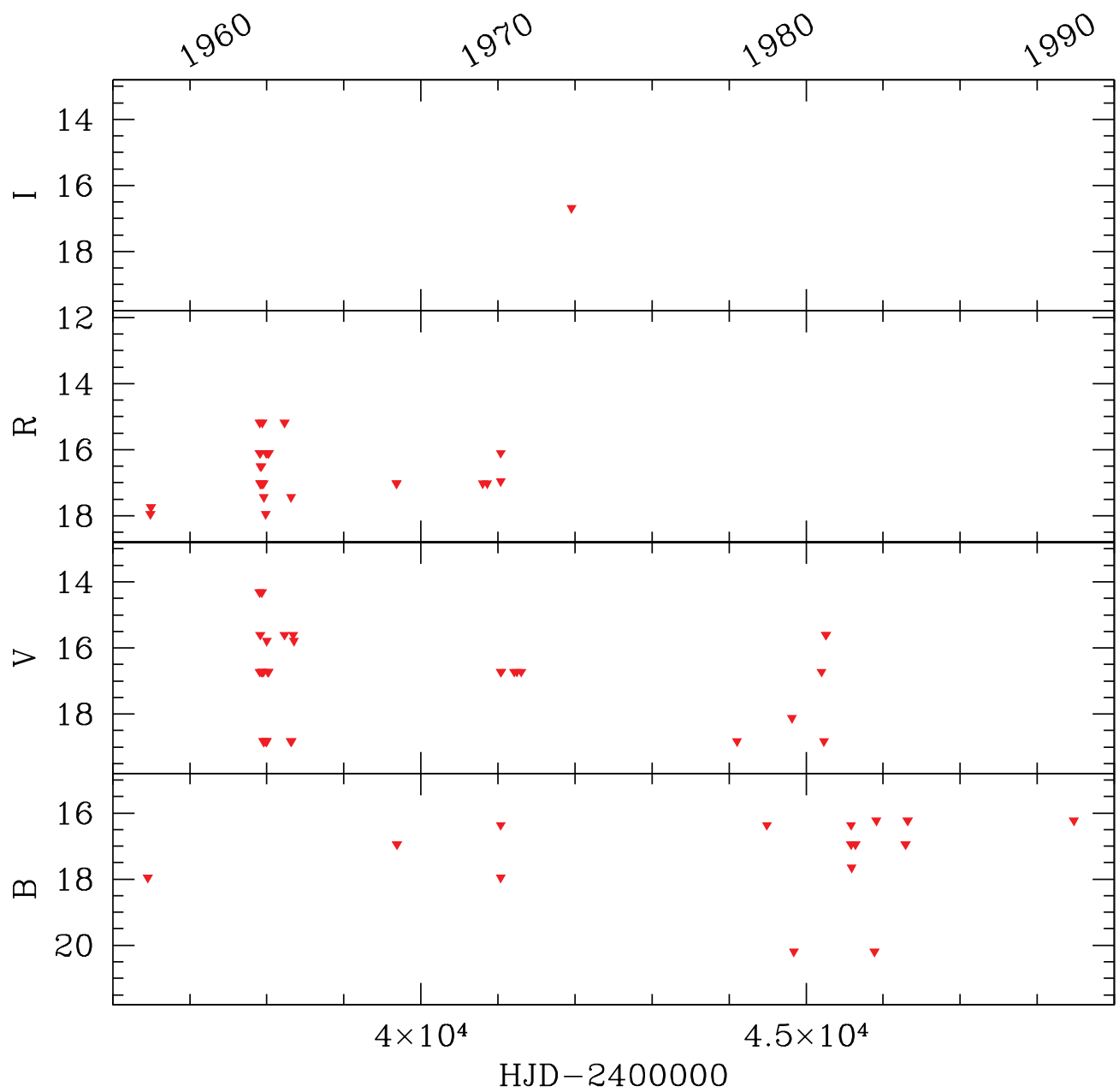

Fig. 1. BVRI magnitude upper limits (solid red triangles) for V2492 Cyg. the framework of FUor and EXor outbursts, and, in principle, even a rapid decrease in the extinction could be responsible for the increased brightness. The $V$-band light curve (2015-2017) from the ASASSN database provides significant upper limits $(V<17 \mathrm{mag}$ ) confirming that ASASSN-15qi is currently in a quiescence period

\section{Data acquisition}

\subsection{Asiago archive plates}

Two Schmidt telescopes were operated with photographic plates at the Astronomical Observatory in Asiago. The smaller one (SP, $40 / 50 \mathrm{~cm}, 100 \mathrm{~cm}$ focal length) observed between 1958 and 1992, with photographic films covering a circular area $5^{\circ}$ in diameter. The larger telescope (SG, 67/92 cm, $208 \mathrm{~cm}$ focal length) exposed square glass photographic plates imaging a $5^{\circ} \times 5^{\circ}$ portion of the sky. It operated with photographic plates between 1965 and 1998, after which large format CCDs were used as detectors. Nearly all plates from both telescopes go very deep, $B \sim 18.5$ and $B \sim 17.8$ mag being the typical limiting magnitude for blue sensitive plates exposed with the SG and SP telescopes, respectively.

The Asiago plates were usually exposed as $103 \mathrm{a}-\mathrm{O}+\mathrm{GG} 13$, 103a-E + RG1, Tri-X + GG14, and IN + RG5 combinations of Kodak plates and Schott astronomical filters, matching the passband of Johnson-Cousins $B, R_{\mathrm{C}}$, and $I_{\mathrm{C}}$ photometric system (Moro \& Munari 2000). A large number of plates were exposed as unfiltered $103 \mathrm{a}-\mathrm{O}$, thus nominally covering both the Johnson
$B$ and $U$ bands thanks to the high ultraviolet transparency of the UBK-7 corrector plates at both Schmidt telescopes. For low temperature and/or reddened objects, especially if they were observed at large airmass, the amount of proper $U$-band photons collected by an unfiltered $103 \mathrm{a}-\mathrm{O}$ plate is however minimal compared to those arriving through the $B$-band portion of the interval of sensitivity of 103a-O emulsion. In such conditions (and provided that the selected comparison stars are themselves of low temperature and/or high reddening), 103a-O + GG13 pairs and unfiltered $103 \mathrm{a}-\mathrm{O}$ plates are almost equally well replicating the standard Johnson $B$ band (Munari \& Dallaporta 2014).

The Asiago Schmidt plates cover an uninterrupted interval of $40 \mathrm{yr}$, and are accurately preserved in a controlled environment. We note that their time spans overlap the so-called Menzel Gap when for about ten years the acquisition of plates with Harvard telescopes distributed around the globe was greatly reduced. A total of 90, 68, and 115 Asiago plates were found to image, respectively, the three program stars V2492 Cyg, V350 Cep and ASASSN-15qi. The resulting magnitudes are listed in Tables A.1-A.3.

\subsection{Harvard plates}

In order to expand our search for old starbursts of our program stars as far back as possible into the past, we turned to the Harvard College Observatory (HCO) plate stack in Cambridge, Massachusetts, home of one of the largest and best preserved archives of astronomical photographic plates taken with many 


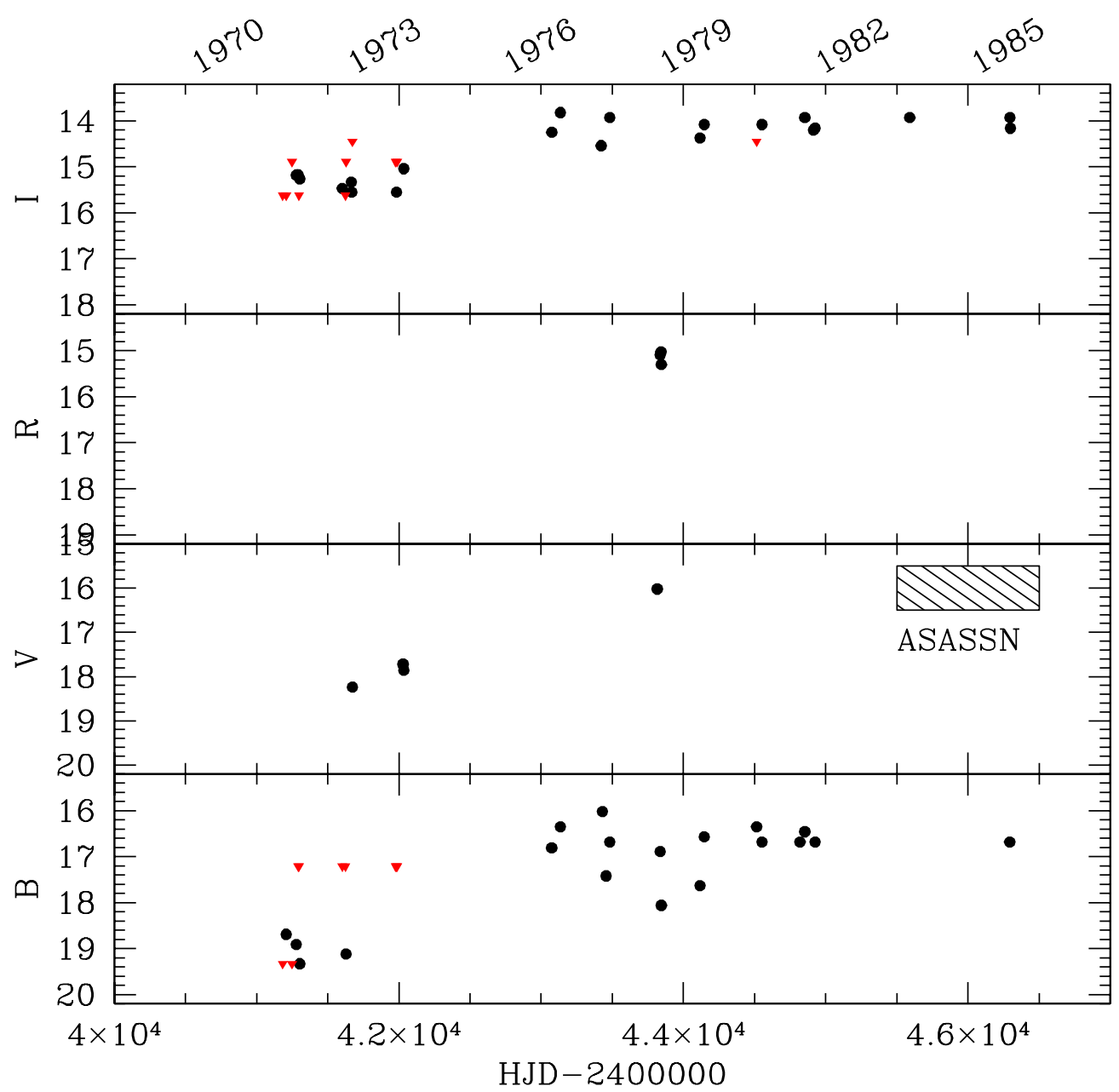

Fig. 2. BVRI light curves of V350 Cep. Upper limits are given as solid red triangles. The hatched box on the right-hand side of the $V$-band panel indicates the spread of magnitudes obtained in the period 2014-2017 by the survey ASASSN (see text). different astrographs located at various sites distributed in both hemispheres beginning around 1880. The limited focal length and aperture of these astrographs result in detection limits which are typically some magnitudes brighter than those of the far deeper Asiago plates.

V350 Cep by lucky coincidence happens to be located in a region of the sky where all $\mathrm{HCO}$ plates have been digitized and measured as part of the DASCH project (Grindlay et al. 2012), that we were allowed to access prior to publication (E. Los, 2017, priv. comm.). The other two targets, V2492 Cyg and ASASSN$15 q \mathrm{q}$, are in regions of the sky not covered by DASCH, so we personally went to the HCO plate stack, located and retrieved the relevant plates, and inspected them at the microscope against local photometric sequences extracted from the all-sky APASS photometric survey (Henden \& Munari 2014). The process is tedious and therefore we decided to limit the inspection to HCO plates from instruments that were expected to pass a limiting magnitude of 13 in $B$ band (almost all of the old HCO plates are blue sensitive with a response comparable to that of modern $B$ band).

About 535 plates for V2492 Cyg and 162 for ASASSN-15qi were selected for visual inspection. About 198 for V2492 Cyg and 40 for ASASSN-15qi were found to be inadequate for the task for a variety of reasons (emulsion defects, fogging, poor guiding/seeing, plate missing or imaging an incorrect field, etc.), leaving 337 suitable plates for V2492 Cyg and 122 for ASASSN15qi. In none of them were the program stars detected. The same holds true for V350 Cep, which is undetected on all plates digitized by DASCH. A random selection of the latter was inspected at the microscope to compare our estimates with DASCH limiting magnitudes, and similar results were found (incidentally, the photometric calibration of DASCH scans is done based on the APASS survey too). Figure 4 presents the upper limits from $\mathrm{HCO}$ plates to the brightness of our program stars in graphical form.

\subsection{Brightness measurement}

To derive the brightness of our targets, we compared them at a high quality Zeiss microscope against a local photometric sequence established around each target. Such a sequence, composed of stars of roughly the same colour as the variable, and widely distributed in magnitude so as to cover both quiescence and outburst states, was extracted primarily from the APASS $B V g^{\prime} r^{\prime} i^{\prime}$ all-sky survey (Henden et al. 2012; Henden \& Munari 2014), with porting to Landolt $R_{\mathrm{C}}$ and $I_{\mathrm{C}}$ bands following Munari (2012) and Munari et al. (2014) prescriptions. To evaluate the measurement errors a number of plates were re-estimated after several days when all memories had vanished from the observer(s). The typical error is $0.1 \mathrm{mag}$, comparable to that intrinsic to the photographic plate itself so that the observer adds little to it. For plates not recording the variable star due to it being too faint, we noted the magnitude of the faintest but still clearly visible star of the comparison sequence. The latter was 


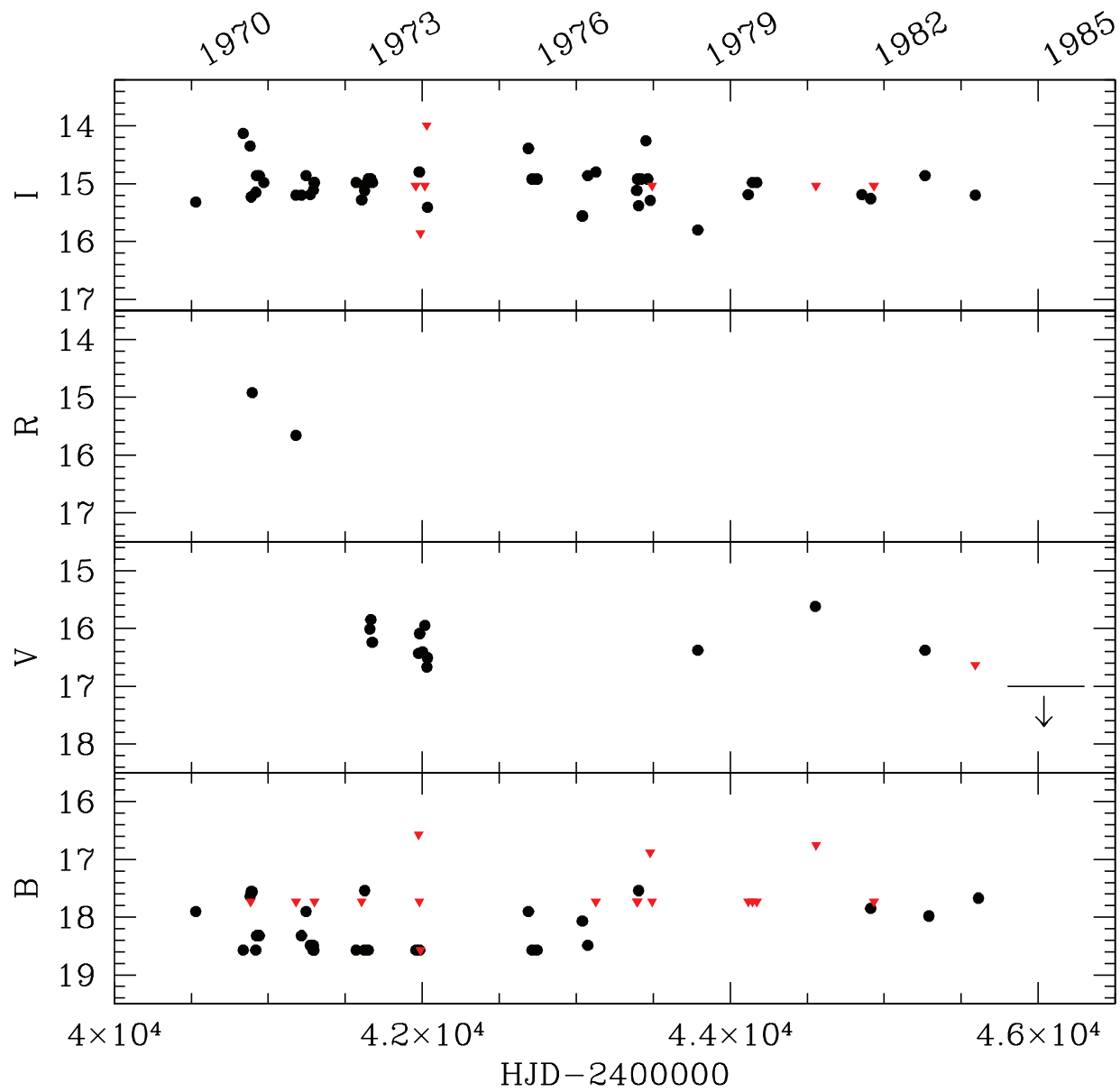

Fig. 3. BVRI light curves of ASASSN 15qi. Upper limits are given as solid red triangles. The horizontal segment in the right side of the $V$-band panel indicates the level of upper limit magnitudes obtained in the period $2015-2017$ by the survey ASASSN (see text). composed by field stars closely distributed around the variable and typically by 0.35 mag in brightness.

\section{Historical light curves}

Because of the limits imposed by the Harvard plate, the analysis presented hereinafter will be based on the Asiago plates only. The BVRI light curves of V2492 Cyg, V350 Cep, and ASASSN 15 qi corresponding to their plate photometry (Tables A.1-A.3) are given in Figs. 1-3, respectively. In Table A.4 some statistics are provided for the source ASASSN-15qi, the only one for which we can infer, for the first time, meaningful averaged values for the quiescence level. Determining this level is fundamental to have a solid reference for accurately computing physical changes once the outburst values are obtained. In each band, we list the number of observations, the median value (basically the quiescence magnitude) together with the standard deviation of the data points, and the peak brightness. We note how this source presents, in quiescence, a level of modest variability quantified by considering the standard deviation of the measurements (0.3-0.4 mag).

\section{Analysis and discussion}

\subsection{V2492 Cyg}

During our monitoring period V2492 Cyg remained always undetected at our sensitivity, therefore the light curve depicted in Fig. 1 displays only upper limit values at different levels, as explained in Sect. 3. As a consequence, no fading or outbursting event can be detected, nevertheless some useful information can be derived. As mentioned above, after its discovery in 2010, V2492 underwent a long-lasting period of strong activity with intermittent burst and fading events (see Hillenbrand et al. 2013 and $\mathrm{AAVSO}^{2}$ data) and reached its maximum recorded brightness in 2017 Giannini et al. (2018). During most of this period, the source was sampled with an almost daily cadence and, for long time intervals, remained brighter than the following values: $B<18, V<16, R<15$, and $I<14$ mag. In comparison, our plate measurements are largely undersampled, presenting long periods (up to a decade) without any data. In any case (not considering the $I$ band, which is practically uncovered, and the $B$ band, which presents no significant upper limits), our $V$ and $R$-band upper limits tentatively suggest that duringa period of about 30 yr from 1958 to 1987 an activity similar (both in duration and in brightness) to that more recently (2010-2017) monitored, did not occur. Indeed, we note that for a significant amount of time the source is brighter than the level indicated by our upper limits, thus suggesting that, in the past, the activity of V2492 Cyg was not as strong as it is now. The above scenario, if correctly described, means that an enhanced brightness variability could be an infrequent feature of V2492 Cyg. Such circumstances favour an accretion- more than an extinctiondriven origin for the bursts. Indeed, the former is expected to occur with a long and irregular cadence related to the viscous motion of the matter toward the inner edge of the disk, while the latter should occur more frequently and regularly, according

\footnotetext{
2 American Association of Variable Star Observers (https://www . aavso.org)
} 


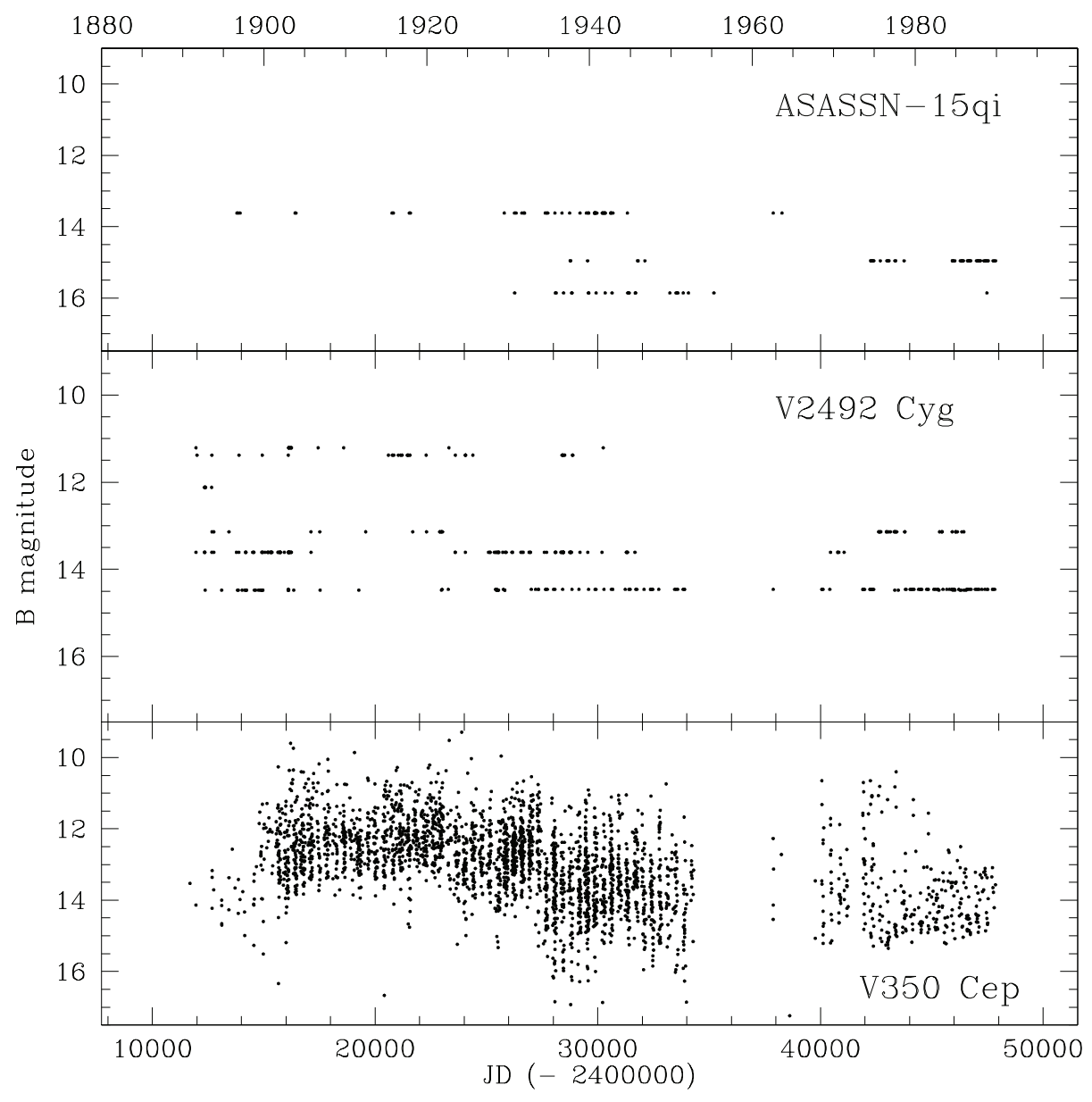

Fig. 4. Limiting magnitude for the Harvard plates inspected in the search for past bright episodes of the program stars. For V2492 Cyg and ASASSN-15qi, we have plotted the $B$-band magnitude of the faintest APASS star visible at the microscope in the immediate surroundings of the targets. For V350 Cep we plot the limiting magnitude as derived by DASCH as part of the calibrations of the plate scans. to the orbital motion of the obscuring matter along the line of sight.

\subsection{V350 Cep}

As depicted in Fig. 2, our 15-year coverage (1971-1985) presents a significant sequence of data in the $B$ and $I$ bands only. These data substantially confirm the behaviour of V350 Cep illustrated in Sect. 1: namely a burst occurred in the middle of the 1970s, after which V350 became well detectable, at a roughly constant level of brightness. To our knowledge, the available literature does not point out any sign of a peculiar activity until the sudden fading and quick restoring of its maximum brightness, occurring in the $B$ band during 2016 (Semkov et al. 2017). Our data indicate this has not been a unique event. Indeed, between 1977 and 1980, in the same band, there is evidence of intermittent luminosity variations of $\Delta B \sim 1-1.5 \mathrm{mag}$ on a time-scale of months. A lesser variation is barely recognisable also in the $I$ band. The existence of (at least) two fading episodes in few decades makes it difficult to classify V350 Cep as a pure FUor star, although its relevant brightening occurred in the 1970s. On the other hand, albeit increasing the monitoring coverage, the lack of significant outbursts remains confirmed, and therefore, also the EXor hypothesis does not seem viable. A longer and continuous multi-band monitoring could provide a more certain classification, but, at the present stage, we note that the presence of an orbiting structure along the line of sight (UXor hypothesis) has a role in determining its light curve. The strict similarity between the two fading episodes highlighted by our observations corroborates such a conclusion.

\section{3. $A S A S S N-15 q i$}

Although much more frequently sampled and for a much longer period, our data substantially agree with the archival photometry given by Herczeg et al. (2016, see their Table 1). Our monitoring refers to a long-lasting (1970-1983) quiescence period during which brightness fluctuations (up to $1 \mathrm{mag}$ ) in the $B, V$, and $I$ bands occurred. Therefore, ASASSN-15qi behaved in the past as a moderately active young source similar to the classical $\mathrm{T}$ Tauri stars, albeit more massive and luminous. It should be noted that a very fast event (lasting about 10 days) analogous to that recently pointed out by Maehara et al. (2015) (see Sect. 1) would, theoretically, never have been detected by our monitoring, whose sampling is not frequent enough. As a consequence, we can only confirm the doubts already expressed by Herczeg et al. (2016) about the classification of this object. However, we can provide an argument related to the colourmagnitude plot (see following Sect. 5.4) not supporting a pure UXor classification.

\subsection{Colour-magnitude diagrams}

Given the available data we can only build the colour-magnitude plots $B$ versus $[B-I]$ for the sources V350 Cep and ASASSN15qi, using only magnitudes obtained within 1 day in $B$ and $I$ bands. They are given in Fig. 5 and essentially support the above considerations. In fact, while data points of V350 Cep (left panel) are well aligned along the extinction vector, ASASSN-15qi (right panel) comparatively shows a more dispersed distribution, typical of sources whose fluctuations cannot be reconciled with a pure extinction origin. 


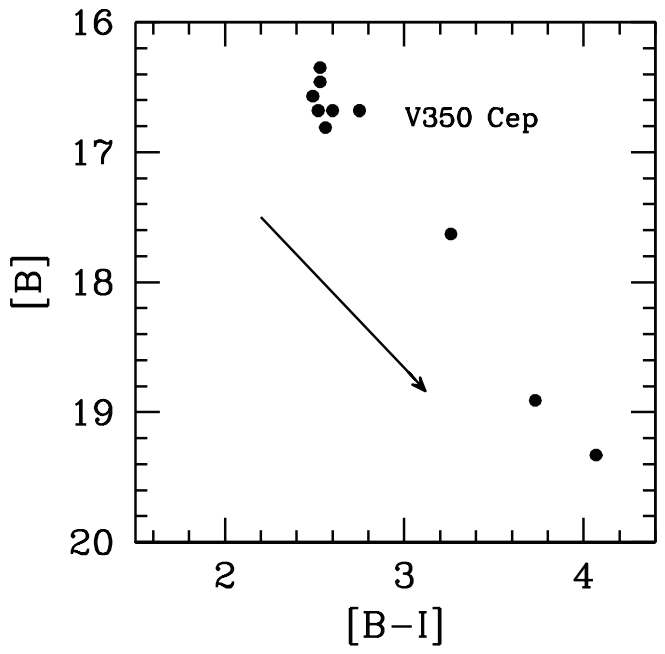

\section{Concluding remarks}

Archival plate analysis is a tool well suited to searching the past history of young variables identified as eruptive stars, and hence to improve their classification. We investigated the Asiago Schmidt plate collection for observations of the selected fields where the three eruptive sources V2492 Cyg, V350 Cep, and ASASSN-15qi are located. Observations of these regions were repeatedly carried out at Asiago over various time periods from 12 to $30 \mathrm{yr}$. The analysis of Harvard plates of the same sources rules out the occurrence of large outbursts in the past. We provide one of the best-sampled photometric datasets ever obtained of the past history of the three targets. In particular, V2492 historical upper limits do not seem compatible with the level of brightness of the present activity. Hence, this latter could have appeared only recently, possibly dominated by accretion phenomena more than by repetitive obscuration. For V350, our monitoring has pointed out that sudden fading followed by rapid restoring of the previous brightness is not a unique and isolated event, and, as such, it may be attributable to an orbiting structure along the line of sight. During the monitored quiescence, ASASSN-15qi presents a level of moderate variability (0.5-1 mag) that is comparable with that of classical $\mathrm{T}$ Tauri stars. We are not able to fill in any gaps existing in the available literature about its nature. We can only say that a pure extinction origin does not seem to be the only mechanism responsible for the observed fluctuations.

Acknowledgements. We acknowledge with thanks the variable star observations from the AAVSO International Database contributed by observers worldwide and used in this research. We thank the ASAS-SN project that is supported by the Gordon and Betty Moore Foundation through grant GBMF5490 to the Ohio State University and NSF grant AST-1515927. We are thankful to the people of the Harvard College Observatory for their kind support. R.J.S. thanks the INAF Astronomical Observatory of Padova for the hospitality during the stay in Asiago and for permission to use the historical plate archive of the Asiago Observatory. This work was supported in part by the Croatian Science Foundation under the project 6212 Solar and Stellar Variability and by the University of Rijeka under the project number 13.12.1.3.03.

\section{References}

Antoniucci, S., Arkharov, A. A., Di Paola, A., et al. 2013, Protostars and Planets VI, 2

Antoniucci, S., Arkharov, A. A., Di Paola, A., et al. 2014, A\&A, 565, L7
Aspin, C. 2011, AJ, 141, 196

Audard, M., Ábrahám, P., Dunham, M. M., et al. 2014, Protostars and Planets VI, 387

Bally, J., \& Reipurth, B. 2003, AJ, 126, 893

Connelley, M. S., Reipurth, B. \& Hillenbrand, L. A. 2015, ATel, 8333

Covey, K. R., Hillenbrand, L. A., Miller, A. A., et al. 2011, AJ, 141, 40

Dahm, S. E., \& Hillenbrand, L. A. 2015, AJ, 149, 200

D'Angelo, C. R., \& Spruit, H. C. 2010, MNRAS, 406, 1208

D’Angelo, C. R., \& Spruit, H. C. 2012, MNRAS, 420, 416

Giannini, T., Lorenzetti, D., Antoniucci, S., et al. 2016, ApJ, 819, L5

Giannini, T., Munari, U., Antoniucci, S., et al. 2018, A\&A, 611, A54

Grindlay, J., Tang, S., Los, E., \& Servillat, M., 2012, IAU Symp., 285, 29

Grinin, V. P. 1988, Sov. Astron. Lett., 14, 27

Hartmann, L., \& Kenyon, S. 1985, ApJ, 299, 462

Hartmann, L., Herczeg, G., \& Calvet, N. 2016 ARAA, 54, 135

Henden, A., \& Munari, U. 2014, CAOSP, 43, 518

Henden, A. A., Levine, S. E., Terrell, D., Smith, T. C., \& Welch, D. 2012, JAAVSO, 40, 430

Herbig, G. H. 1989, in Proc. of the ESO Workshop on Low Mass Star Formation and Pre-Main Sequence Objects, ed. B. Reipurth, 233

Herbig, G. H. 2008, AJ, 135, 637

Herczeg, G. J., Dong, S., Shappee, B. J., et al. 2016. ApJ, 831, 133

Hillenbrand, L. A., Miller, A. A., Covey, K. R. et al. 2013, AJ, 145, 59

Hillenbrand, L. A., Reipurth, B., \& Connelley, M. S. 2015, ATel, 8331

Ibryanov, S., \& Semkov, E. 2017, ATel, 10170

Ibryanov, S., Semkov, E., \& Peneva, S. 2014, Res. Astron. Astrophys., 14, 1264

Itagaki, K., \& Yamaoka, H. 2010, CBET, 2426, 1

Jurdana-Šepić, R., \& Munari, U. 2016, New Astron., 43, 87

Jurdana-Šepić, R., Munari, U., Antoniucci, S., et al. 2017, A\&A, 602, A99

Kóspál, Á., Ábrahám, P., Acosta-Pulido, J. A., et al. 2011, A\&A, 527, A133

Kóspál, Á., Ábrahám, P., Acosta-Pulido, J. A., et al. 2013, A\&A, 551, A62

Kóspál, Á., Ábrahám, P., Westhues, Ch. \& Haas, M. 2017, A\&A, 597, L10

Kraus, S., Caratti o Garatti, A., Garcia-Lopez, R., et al. 2016, MNRAS, 462, L61

Lorenzetti, D., Larionov, V. M., Giannini, T., et al. 2009, ApJ, 693, 1056

Lorenzetti, D., Antoniucci, S., Giannini, T., et al. 2012, ApJ, 749, 188

Maehara, H., Ayani, K., Itoh, R., Takata, K., \& Kawabata, K. S. 2015, ATel, 8147

Moro, D., \& Munari, U. 2000, A\&AS, 147, 361

Munari, U., 2012, JAVSO, 40, 582

Munari, U., \& Dallaporta, S. 2014, New Astron., 27, 25

Munari, U., Henden, A., Frigo, A., et al. 2014, AJ, 148, 81

Munari, U., Traven, G., Dallaporta, S., et al. 2017, ATel, 10183

Pogosyants, A. Yu. 1991, IBVS 3624, 1

Rieke, G. H., \& Lebofsky, M. J. 1985, ApJ, 288, 618

Semkov, E. H. 2004, IBVS, 5556, 1

Semkov, E. H., Ibryamov, S. I., \& Peneva, S. P. 2017, Bulg. Astron. J., 27, 75

Sicilia-Aguilar, A., Merín, B., Hormuth, F., et al. 2008, ApJ, 673, 382

Sicilia-Aguilar, A., Kóspál, Á., Setiawan, J., et al. 2012, A\&A, 544, A93

Straižys, V., Maskoliūnas, M., Boyle, R. P., et al. 2014, MNRAS, 438, 1848

Zhu, Z., Hartmann, L., \& Gammie, C., 2009, ApJ, 694, 1045

Xiao, L., Kroll, P., \& Henden, A. A. 2010, AJ, 139, 1527 
Appendix A

Table A.1. Plate photometry of V2492 Cyg.

\begin{tabular}{|c|c|c|c|c|c|c|c|c|}
\hline HJD & Date & UT & Expt & Emulsion & Filter & Tel & Plate & mag \\
\hline 2436454.42875 & 1958-09-07 & $22: 13$ & 30 & $103 \mathrm{a}-\mathrm{O}$ & .. & SP & 00031 & $B>17.95$ \\
\hline 2436488.31134 & 1958-10-11 & $19: 25$ & 60 & $103 a-E$ & RG 1 & $\mathrm{SP}$ & 00049 & $R>17.95$ \\
\hline 2436494.29375 & 1958-10-17 & 19:00 & 40 & 103 a-E & RG 1 & SP & 00052 & $R>17.74$ \\
\hline 2437905.46837 & $1962-08-28$ & $23: 10$ & 15 & PANROY & $\ldots$. & SP & 03028 & $V>14.32$ \\
\hline 2437907.44614 & $1962-08-30$ & $22: 38$ & 15 & PANROY & $\ldots$ & $\mathrm{SP}$ & 03037 & $V>16.72$ \\
\hline 2437907.46420 & $1962-08-30$ & 23:04 & 15 & 103 a-E & RG 1 & SP & 03038 & $R>15.18$ \\
\hline 2437908.48295 & 1962-08-31 & $23: 31$ & 40 & 103 a-E & RG 1 & SP & 03045 & $R>16.10$ \\
\hline 2437913.51001 & 1962-09-05 & $24: 10$ & 15 & PANROY & $\ldots$. & SP & 03055 & $V>15.60$ \\
\hline 2437913.55098 & 1962-09-05 & $25: 09$ & 30 & $103 \mathrm{a}-\mathrm{E}$ & RG 1 & SP & 03056 & $R>16.10$ \\
\hline 2437915.47180 & 1962-09-07 & $23: 15$ & 30 & 103 a-E & RG 1 & $\mathrm{SP}$ & 03060 & $R>17.02$ \\
\hline 2437916.44819 & 1962-09-08 & $22: 41$ & 40 & 103 a-E & RG 1 & SP & 03065 & $R>17.02$ \\
\hline 2437917.48776 & 1962-09-09 & $23: 38$ & 40 & $103 \mathrm{a}-\mathrm{E}$ & RG 1 & SP & 03075 & $R>16.50$ \\
\hline 2437926.33139 & $1962-09-18$ & $19: 53$ & 30 & 103 a-E & RG 1 & SP & 03083 & $R>17.02$ \\
\hline 2437929.32509 & 1962-09-21 & $19: 44$ & 40 & $103 \mathrm{a}-\mathrm{E}$ & RG 1 & SP & 03095 & $R>16.50$ \\
\hline 2437930.37645 & $1962-09-22$ & $20: 58$ & 15 & PANROY & $\ldots$ & SP & 03101 & $V>16.72$ \\
\hline 2437933.46666 & $1962-09-25$ & $23: 08$ & 40 & 103 a-E & RG 1 & SP & 03118 & $R>17.02$ \\
\hline 2437936.52909 & $1962-09-28$ & $24: 38$ & 15 & PANROY & $\ldots$ & SP & 03133 & $V>14.32$ \\
\hline 2437938.42557 & 1962-09-30 & 22:09 & 15 & PANROY & $\ldots$. & $\mathrm{SP}$ & 03146 & $V>16.72$ \\
\hline 2437939.40401 & $1962-10-01$ & $21: 38$ & 15 & PANROY & $\ldots$ & SP & 03161 & $V>16.72$ \\
\hline 2437940.35468 & 1962-10-02 & $20: 27$ & 40 & 103 a-E & RG 1 & $\mathrm{SP}$ & 03170 & $R>17.02$ \\
\hline 2437942.37337 & 1962-10-04 & $20: 54$ & 40 & 103 a-E & RG 1 & SP & 03189 & $R>15.18$ \\
\hline 2437955.28264 & $1962-10-17$ & $18: 44$ & 15 & PANROY & $\ldots$ & SP & 03208 & $V>16.72$ \\
\hline 2437956.29024 & 1962-10-18 & $18: 55$ & 30 & 103 a-E & RG 1 & SP & 03213 & $R>17.02$ \\
\hline 2437957.28742 & $1962-10-19$ & $18: 51$ & 20 & PANROY & $\ldots$ & SP & 03219 & $V>18.82$ \\
\hline 2437960.28591 & $1962-10-22$ & $18: 49$ & 40 & 103 a-E & RG 1 & SP & 03225 & $R>17.43$ \\
\hline 2437961.27892 & $1962-10-23$ & $18: 39$ & 20 & PANROY & $\ldots$. & SP & 03240 & $V>18.82$ \\
\hline 2437962.30805 & $1962-10-24$ & $19: 21$ & 20 & PANROY & $\ldots$ & SP & 03249 & $V>18.82$ \\
\hline 2437985.23194 & $1962-11-16$ & $17: 33$ & 40 & $103 a-E$ & RG 1 & $\mathrm{SP}$ & 03294 & $R>17.95$ \\
\hline 2437991.24829 & $1962-11-22$ & $17: 57$ & 20 & PANROY & $\ldots$. & SP & 03298 & $V>18.82$ \\
\hline 2437992.23296 & $1962-11-23$ & $17: 35$ & 40 & 103 a-E & RG 1 & SP & 03311 & $R>16.10$ \\
\hline 2437995.21822 & $1962-11-26$ & $17: 14$ & 20 & PANROY & $\ldots$ & SP & 03337 & $V>18.82$ \\
\hline 2437998.21251 & $1962-11-29$ & $17: 06$ & 15 & PANROY & $\ldots$ & SP & 03347 & $V>18.82$ \\
\hline 2437999.23328 & $1962-11-30$ & $17: 36$ & 40 & 103 a-E & RG 1 & $\mathrm{SP}$ & 03367 & $R>16.10$ \\
\hline 2438000.23601 & 1962-12-01 & $17: 40$ & 10 & PANROY & $\ldots$. & $\mathrm{SP}$ & 03382 & $V>15.78$ \\
\hline 2438013.25477 & $1962-12-14$ & $18: 08$ & 15 & PANROY & $\ldots$ & $\mathrm{SP}$ & 03440 & $V>16.72$ \\
\hline 2438015.21369 & $1962-12-16$ & $17: 09$ & 30 & $103 \mathrm{a}-\mathrm{E}$ & RG 1 & SP & 03447 & $R>16.10$ \\
\hline 2438017.21429 & $1962-12-18$ & $17: 10$ & 30 & 103 a-E & RG 1 & SP & 03466 & $R>16.10$ \\
\hline 2438019.23502 & $1962-12-20$ & $17: 40$ & 15 & PANROY & $\ldots$. & SP & 03483 & $V>16.72$ \\
\hline 2438020.24677 & $1962-12-21$ & $17: 57$ & 30 & 103 a-E & RG 1 & $\mathrm{SP}$ & 03493 & $R>16.10$ \\
\hline 2438021.21201 & $1962-12-22$ & $17: 07$ & 15 & PANROY & $\ldots$ & SP & 03507 & $V>16.72$ \\
\hline 2438024.21394 & $1962-12-25$ & $17: 10$ & 30 & $103 a-E$ & RG 1 & SP & 03525 & $R>16.10$ \\
\hline 2438229.49955 & 1963-07-18 & $23: 56$ & 10 & PANROY & $\ldots$ & SP & 04082 & $V>15.60$ \\
\hline 2438230.50514 & 1963-07-19 & $24: 04$ & 30 & $103 \mathrm{a}-\mathrm{E}$ & RG 1 & $\mathrm{SP}$ & 04091 & $R>15.18$ \\
\hline 2438311.30172 & $1963-10-08$ & $19: 11$ & 13 & PANROY & $\ldots$ & SP & 04095 & $V>18.82$ \\
\hline 2438314.31482 & $1963-10-11$ & $19: 30$ & 30 & 103 a-E & RG 1 & $\mathrm{SP}$ & 04108 & $R>17.43$ \\
\hline 2438321.30761 & $1963-10-18$ & $19: 20$ & 22 & PANROY & $\ldots$ & $\mathrm{SP}$ & 04150 & $V>18.82$ \\
\hline 2438342.26500 & 1963-11-08 & $18: 20$ & 15 & PANROY & $\ldots$ & SP & 04229 & $V>15.60$ \\
\hline 2438351.24371 & 1963-11-17 & $17: 50$ & 15 & PANROY & $\ldots$ & SP & 04238 & $V>15.78$ \\
\hline 2439681.53672 & 1967-07-09 & $24: 50$ & 60 & $103 a-E$ & RG 1 & SG & 00741 & $R>17.02$ \\
\hline 2439685.47923 & $1967-07-13$ & $23: 27$ & 90 & $103 a-E$ & RG 1 & SG & 00754 & $R>17.02$ \\
\hline 2439688.53768 & 1967-07-16 & $24: 51$ & 30 & $0 \mathrm{a}-\mathrm{O}$ & & SG & 00768 & $B>16.95$ \\
\hline
\end{tabular}

Notes. Columns provide: date and central UT of any exposure, exposure time, plate emulsion, the adopted filter, the telescope, the plate number, and the magnitude derived for the source (see text for further details). 
Table A.1. continued.

\begin{tabular}{|c|c|c|c|c|c|c|c|c|}
\hline HJD & Date & UT & Expt & Emulsion & Filter & Tel & Plate & mag \\
\hline 2440802.49793 & $1970-08-03$ & $23: 53$ & 60 & Ia-E & RG 6450 & SG & 03527 & $R>17.02$ \\
\hline 2440859.42767 & $1970-09-29$ & $22: 12$ & 60 & $103 a-E$ & RG 6450 & SG & 03654 & $R>17.02$ \\
\hline 2441035.62019 & $1971-03-24$ & $26: 57$ & 15 & $103 \mathrm{a}-\mathrm{O}$ & GG 13 & SP & 08717 & $B>16.36$ \\
\hline 2441035.62575 & $1971-03-24$ & $27: 05$ & 10 & $103 a-O$ & GG 13 & SG & 04262 & $B>17.95$ \\
\hline 2441035.63616 & $1971-03-24$ & $27: 20$ & 10 & 103 a-E & RG 1 & SP & 08718 & $R>16.95$ \\
\hline 2441035.63686 & $1971-03-24$ & $27: 21$ & 10 & $103 a-D$ & GG 14 & SG & 04263 & $V>16.72$ \\
\hline 2441035.65144 & $1971-03-24$ & $27: 42$ & 20 & $103 a-E$ & RG 1 & SG & 04264 & $R>16.10$ \\
\hline 2441040.64879 & $1971-03-29$ & $27: 38$ & 15 & $103 a-D$ & GG 14 & SG & 04281 & $V>16.72$ \\
\hline 2441213.40778 & 1971-09-18 & $21: 43$ & 20 & TRI X & GG 14 & SP & 09022 & $V>16.72$ \\
\hline 2441243.45344 & $1971-10-18$ & $22: 50$ & 20 & TRI X & GG 14 & SP & 09122 & $V>16.72$ \\
\hline 2441303.29768 & $1971-12-17$ & $19: 10$ & 20 & TRI X & GG 14 & SP & 09312 & $V>16.72$ \\
\hline 2441954.46936 & $1973-09-28$ & $23: 12$ & 20 & IN Sen & RG 5 & SG & 06655 & $I>16.69$ \\
\hline 2444101.43147 & $1979-08-15$ & $22: 17$ & 30 & 103a-D & GG 14 & SG & 10133 & $V>18.82$ \\
\hline 2444490.47666 & 1980-09-07 & $23: 22$ & 8 & $103 \mathrm{a}-\mathrm{O}$ & GG 13 & SP & 14349 & $B>16.36$ \\
\hline 2444814.50196 & $1981-07-28$ & $23: 59$ & 30 & $103 a-D$ & GG 14 & SG & 11050 & $V>18.12$ \\
\hline 2444841.49961 & $1981-08-24$ & $23: 55$ & 30 & $103 \mathrm{a}-\mathrm{O}$ & GG 13 & SG & 11084 & $B>20.19$ \\
\hline 2445199.48844 & $1982-08-17$ & $23: 39$ & 15 & TRI X & .... & SP & 14906 & $V>16.72$ \\
\hline 2445231.39041 & 1982-09-18 & $21: 18$ & 30 & 103a-D & GG 14 & SG & 11682 & $V>18.82$ \\
\hline 2445258.38896 & $1982-10-15$ & $21: 17$ & 15 & TRIX & $\ldots$ & SP & 14982 & $V>15.60$ \\
\hline 2445259.39656 & $1982-10-16$ & $21: 28$ & 15 & TRIX & $\ldots$ & SP & 14994 & $V>15.60$ \\
\hline 2445582.38849 & $1983-09-04$ & $21: 15$ & 15 & $103 \mathrm{a}-\mathrm{O}$ & GG 13 & $\mathrm{SP}$ & 15354 & $B>16.95$ \\
\hline 2445583.38015 & $1983-09-05$ & $21: 03$ & 15 & $103 \mathrm{a}-\mathrm{O}$ & GG 13 & SP & 15361 & $B>16.36$ \\
\hline 2445590.41065 & $1983-09-12$ & $21: 47$ & 10 & $103 \mathrm{a}-\mathrm{O}$ & $\ldots$ & $\mathrm{SP}$ & 15380 & $B>17.65$ \\
\hline 2445641.38819 & 1983-11-02 & $21: 17$ & 12 & $103 \mathrm{a}-\mathrm{O}$ & $\ldots$ & SP & 15480 & $B>16.95$ \\
\hline 2445886.49766 & $1984-07-04$ & $23: 54$ & 30 & $103 \mathrm{a}-\mathrm{O}$ & GG 13 & SG & 12519 & $B>20.19$ \\
\hline 2445912.46799 & $1984-07-30$ & $23: 10$ & 10 & $103 \mathrm{a}-\mathrm{O}$ & .... & SP & 15930 & $B>16.23$ \\
\hline 2446290.50297 & $1985-08-12$ & $24: 00$ & 12 & $103 a-O$ & $\ldots$. & SP & 16379 & $B>16.95$ \\
\hline 2446318.42526 & $1985-09-09$ & $22: 08$ & 10 & $103 \mathrm{a}-\mathrm{O}$ & $\ldots$ & SP & 16461 & $B>16.23$ \\
\hline 2448476.44454 & 1991-08-07 & $22: 36$ & 10 & $103 \mathrm{a}-\mathrm{O}$ & GG 13 & SP & 18248 & $B>16.23$ \\
\hline 2448539.46904 & 1991-10-09 & $23: 12$ & 8 & $103 \mathrm{a}-\mathrm{O}$ & GG 13 & $\mathrm{SP}$ & 18303 & $B>16.36$ \\
\hline
\end{tabular}


A\&A 614, A9 (2018)

Table A.2. As in Table A.1, for V350 Cep.

\begin{tabular}{|c|c|c|c|c|c|c|c|c|}
\hline HJD & date & UT & expt & emulsion & filter & tel & plate & mag \\
\hline 2441183.55876 & $1971-08-19$ & $25: 23$ & 20 & $103 a-O$ & $\ldots$ & $\mathrm{SG}$ & 04613 & $B>19.33$ \\
\hline 2441183.58515 & 1971-08-19 & $26: 01$ & 20 & $\mathrm{I}-\mathrm{N}$ sen & RG 5 & SG & 04614 & $I>15.62$ \\
\hline 2441207.40035 & $1971-09-12$ & $21: 34$ & 20 & $103 a-O$ & GG 13 & $\mathrm{SG}$ & 04619 & $B=18.69$ \\
\hline 2441207.45521 & 1971-09-12 & $22: 53$ & 30 & $\mathrm{I}-\mathrm{N}$ sen & RG 5 & $\mathrm{SG}$ & 04621 & $I>15.62$ \\
\hline 2441248.39933 & $1971-10-23$ & $21: 32$ & 20 & $103 a-O$ & GG 13 & $\mathrm{SG}$ & 04827 & $B>19.33$ \\
\hline 2441248.42086 & $1971-10-23$ & $22: 03$ & 30 & $\mathrm{I}-\mathrm{N}$ sen & RG 5 & $\mathrm{SG}$ & 04828 & $I>14.89$ \\
\hline 2441279.28642 & $1971-11-23$ & $18: 50$ & 30 & $\mathrm{I}-\mathrm{N}$ sen & RG 5 & $\mathrm{SG}$ & 04967 & $I=15.18$ \\
\hline 2441279.35239 & $1971-11-23$ & $20: 25$ & 20 & $103 a-O$ & GG 13 & $\mathrm{SG}$ & 04970 & $B=18.91$ \\
\hline 2441293.26107 & $1971-12-07$ & $18: 14$ & 30 & $\mathrm{I}-\mathrm{N}$ sen & RG 5 & $\mathrm{SG}$ & 05013 & $I=15.18$ \\
\hline 2441293.28121 & $1971-12-07$ & $18: 43$ & 20 & Ia-O & GG 13 & $\mathrm{SG}$ & 05014 & $B>17.21$ \\
\hline 2441298.30883 & $1971-12-12$ & $19: 23$ & 20 & Ia-O & GG 13 & $\mathrm{SG}$ & 05068 & $B>17.21$ \\
\hline 2441298.32897 & $1971-12-12$ & $19: 52$ & 30 & $\mathrm{I}-\mathrm{N}$ sen & RG 5 & SG & 05069 & $I>15.62$ \\
\hline 2441303.26285 & $1971-12-17$ & $18: 17$ & 30 & $\mathrm{I}-\mathrm{N}$ sen & RG 5 & $\mathrm{SG}$ & 05117 & $I=15.26$ \\
\hline 2441303.28784 & $1971-12-17$ & $18: 53$ & 20 & $103 a-O$ & GG 13 & SG & 05118 & $B=19.33$ \\
\hline 2441601.46877 & $1972-10-10$ & $23: 12$ & 20 & $103 \mathrm{a}-\mathrm{O}$ & GG 13 & $\mathrm{SG}$ & 05665 & $B>17.21$ \\
\hline 2441601.49169 & $1972-10-10$ & $23: 45$ & 30 & $\mathrm{I}-\mathrm{N}$ sen & RG 5 & $\mathrm{SG}$ & 05666 & $I=15.47$ \\
\hline 2441624.30411 & $1972-11-02$ & $19: 15$ & 20 & $103 a-O$ & GG 13 & $\mathrm{SG}$ & 05746 & $B>17.21$ \\
\hline 2441624.32495 & $1972-11-02$ & $19: 45$ & 30 & $\mathrm{I}-\mathrm{N}$ sen & RG 5 & $\mathrm{SG}$ & 05747 & $I>15.62$ \\
\hline 2441628.29157 & $1972-11-06$ & $18: 57$ & 20 & $103 a-O$ & GG 13 & $\mathrm{SG}$ & 05806 & $B=19.12$ \\
\hline 2441628.31240 & 1972-11-06 & $19: 27$ & 30 & $\mathrm{I}-\mathrm{N}$ sen & RG 5 & $\mathrm{SG}$ & 05807 & $I>14.89$ \\
\hline 2441668.22049 & $1972-12-16$ & $17: 16$ & 25 & I-N sen & RG 5 & $\mathrm{SG}$ & 05955 & $I=15.55$ \\
\hline 2441665.43030 & $1972-12-13$ & $22: 18$ & 05 & $\mathrm{I}-\mathrm{N}$ sen & RG 5 & $\mathrm{SG}$ & 06003 & $I=15.33$ \\
\hline 2441674.21960 & $1972-12-22$ & $17: 15$ & 30 & $\mathrm{I}-\mathrm{N}$ sen & RG 5 & $\mathrm{SG}$ & 06015 & $I>14.45$ \\
\hline 2441674.23974 & $1972-12-22$ & $17: 44$ & 20 & $103 a-D$ & GG 14 & $\mathrm{SG}$ & 06016 & $V=18.24$ \\
\hline 2441977.49586 & $1973-10-21$ & $23: 51$ & 20 & $103 a-O$ & GG 13 & $\mathrm{SG}$ & 06739 & $B>17.21$ \\
\hline 2441977.51461 & $1973-10-21$ & $24: 18$ & 30 & I-N sen & RG 5 & $\mathrm{SG}$ & 06740 & $I>14.89$ \\
\hline 2441983.33958 & $1973-10-27$ & $20: 06$ & 30 & $\mathrm{I}-\mathrm{N}$ sen & RG 5 & $\mathrm{SG}$ & 06800 & $I=15.55$ \\
\hline 2441983.36181 & $1973-10-27$ & $20: 38$ & 20 & $103 a-O$ & GG 13 & SG & 06801 & $B>17.21$ \\
\hline 2441988.42565 & 1973-11-01 & $22: 10$ & 30 & $\mathrm{I}-\mathrm{N}$ sen & RG 5 & $\mathrm{SG}$ & 06854 & $I>14.89$ \\
\hline 2441988.44996 & $1973-11-01$ & $22: 45$ & 20 & $103 a-O$ & GG 13 & SG & 06855 & $B>17.21$ \\
\hline 2442029.21438 & $1973-12-12$ & $17: 07$ & 30 & 103a-D & GG 14 & $\mathrm{SG}$ & 06922 & $V=17.72$ \\
\hline 2442034.26005 & $1973-12-17$ & $18: 13$ & 20 & $103 a-D$ & GG 14 & $\mathrm{SG}$ & 06945 & $V=17.86$ \\
\hline 2442034.28088 & $1973-12-17$ & $18: 43$ & 30 & $\mathrm{I}-\mathrm{N}$ sen & RG 5 & $\mathrm{SG}$ & 06946 & $I=15.04$ \\
\hline 2443074.36738 & $1976-10-22$ & $20: 46$ & 30 & $103 a-O$ & GG 13 & $\mathrm{SG}$ & 08724 & $B=16.81$ \\
\hline 2443074.42225 & $1976-10-22$ & $22: 05$ & 30 & I-N sen & RG 5 & $\mathrm{SG}$ & 08727 & $I=14.25$ \\
\hline 2443135.31196 & $1976-12-22$ & $19: 28$ & 30 & $103 \mathrm{a}-\mathrm{O}$ & GG 13 & $\mathrm{SG}$ & 08818 & $B=16.35$ \\
\hline 2443135.33696 & $1976-12-22$ & $20: 04$ & 30 & $\mathrm{I}-\mathrm{N}$ sen & RG 5 & $\mathrm{SG}$ & 08819 & $I=13.82$ \\
\hline 2443421.44929 & 1977-10-04 & $22: 44$ & 30 & $\mathrm{I}-\mathrm{N}$ sen & RG 5 & $\mathrm{SG}$ & 09235 & $I=14.54$ \\
\hline 2443430.50003 & $1977-10-13$ & $23: 57$ & 20 & $103 a-O$ & GG 13 & $\mathrm{SG}$ & 09252 & $B=16.02$ \\
\hline 2443430.52364 & $1977-10-13$ & $24: 31$ & 30 & $\mathrm{I}-\mathrm{N}$ sen & RG 5 & $\mathrm{SG}$ & 09253 & $I=14.01$ \\
\hline 2443456.49987 & 1977-11-08 & $23: 57$ & 30 & $\mathrm{I}-\mathrm{N}$ sen & RG 5 & SG & 09292 & $I=14.37$ \\
\hline 2443456.52279 & $1977-11-08$ & $24: 30$ & 20 & $103 \mathrm{a}-\mathrm{O}$ & GG 13 & $\mathrm{SG}$ & 09293 & $B=17.42$ \\
\hline 2443482.25558 & $1977-12-04$ & $18: 06$ & 30 & $103 \mathrm{a}-\mathrm{O}$ & GG 13 & $\mathrm{SG}$ & 09352 & $B=16.68$ \\
\hline 2443482.28058 & $1977-12-04$ & $18: 42$ & 30 & I-N sen & RG 5 & $\mathrm{SG}$ & 09353 & $I=13.93$ \\
\hline 2443816.39439 & $1978-11-03$ & $21: 25$ & 30 & $103 a-D$ & GG 14 & $\mathrm{SG}$ & 09723 & $V=16.02$ \\
\hline 2443837.31348 & $1978-11-24$ & $19: 29$ & 20 & $103 \mathrm{a}-\mathrm{O}$ & GG 13 & $\mathrm{SG}$ & 09793 & $B=16.89$ \\
\hline 2443837.33500 & $1978-11-24$ & $20: 00$ & 30 & $103 a-E$ & RG 1 & $\mathrm{SG}$ & 09794 & $R=15.09$ \\
\hline 2443842.35919 & $1978-11-29$ & $20: 35$ & 30 & $103 a-E$ & RG 1 & $\mathrm{SG}$ & 09804 & $R=15.02$ \\
\hline 2443845.35008 & $1978-12-02$ & $20: 22$ & 30 & $103 a-E$ & RG 1 & $\mathrm{SG}$ & 09825 & $R=15.30$ \\
\hline 2443845.37231 & $1978-12-02$ & $20: 54$ & 20 & $103 a-O$ & GG 13 & $\mathrm{SG}$ & 09826 & $B=18.06$ \\
\hline 2444116.51532 & $1979-08-30$ & $24: 20$ & 20 & $103 a-O$ & GG 13 & $\mathrm{SG}$ & 10164 & $B=17.63$ \\
\hline 2444116.53755 & $1979-08-30$ & $24: 52$ & 30 & $\mathrm{I}-\mathrm{N}$ sen & RG 5 & $\mathrm{SG}$ & 10165 & $I=14.37$ \\
\hline 2444146.36243 & $1979-09-29$ & $20: 39$ & 30 & I-N sen & RG 5 & $\mathrm{SG}$ & 10197 & $I=14.08$ \\
\hline 2444146.39090 & 1979-09-29 & $21: 20$ & 20 & $103 a-O$ & GG 13 & $\mathrm{SG}$ & 10198 & $B=16.57$ \\
\hline 2444514.28954 & $1980-10-01$ & $18: 54$ & 30 & $\mathrm{I}-\mathrm{N}$ sen & RG 5 & $\mathrm{SG}$ & 10629 & $I>14.45$ \\
\hline 2444514.33746 & 1980-10-01 & 20:03 & 30 & $103 a-O$ & GG 13 & $\mathrm{SG}$ & 10630 & $B=16.35$ \\
\hline
\end{tabular}


R. Jurdana-Šepić et al.: Towards a better classification of unclear eruptive variables

Table A.2. continued.

\begin{tabular}{cccclllll}
\hline \hline HJD & date & UT & expt & emulsion & filter & tel & plate & mag \\
\hline 2444551.49850 & $1980-11-07$ & $23: 55$ & 20 & 103a-O & GG 13 & SG & 10662 & $B=16.68$ \\
2444551.52072 & $1980-11-07$ & $24: 27$ & 30 & I-N sen & RG 5 & SG & 10663 & $I=14.08$ \\
2444819.49780 & $1981-08-02$ & $23: 56$ & 30 & 103a-O & GG 13 & SG & 11056 & $B=16.68$ \\
2444853.32451 & $1981-09-05$ & $19: 45$ & 30 & I-N sen & RG 5 & SG & 11089 & $I=13.93$ \\
2444853.35090 & $1981-09-05$ & $20: 23$ & 30 & 103a-O & GG 13 & SG & 11090 & $B=16.46$ \\
2444913.36104 & $1981-11-04$ & $20: 37$ & 30 & I-N sen & RG 5 & SG & 11168 & $I=14.20$ \\
2444926.33862 & $1981-11-17$ & $20: 05$ & 30 & I-N sen & RG 5 & SG & 11191 & $I=14.16$ \\
2444926.36292 & $1981-11-17$ & $20: 40$ & 30 & 103a-O & GG 13 & SG & 11192 & $B=16.68$ \\
2445591.38996 & $1983-09-13$ & $21: 19$ & 30 & I-N sen & RG 5 & SG & 12208 & $I=13.93$ \\
2446293.45032 & $1985-08-15$ & $22: 47$ & 30 & IIa-O & GG 13 & SG & 12930 & $B=16.68$ \\
2446295.46427 & $1985-08-17$ & $23: 07$ & 30 & I-N sen & RG 5 & SG & 12939 & $I=13.93$ \\
2446297.44280 & $1985-08-19$ & $22: 36$ & 30 & I-N sen & RG 5 & SG & 12948 & $I=14.16$ \\
\hline
\end{tabular}


A\&A 614, A9 (2018)

Table A.3. As in Table A.1, for ASASSN-15qi.

\begin{tabular}{|c|c|c|c|c|c|c|c|c|}
\hline HJD & date & UT & expt & emulsion & filter & tel & plate & mag \\
\hline 2440529.26267 & 1969-11-03 & $18: 14$ & 30 & $103 \mathrm{a}-\mathrm{O}$ & GG 13 & SG & 02750 & $B=17.90$ \\
\hline 2440529.28697 & 1969-11-03 & $18: 49$ & 30 & $\mathrm{I}-\mathrm{N}$ sen & RG 5 & SG & 02751 & $I=15.32$ \\
\hline 2440836.43372 & 1970-09-06 & $22: 21$ & 30 & $103 \mathrm{a}-\mathrm{O}$ & GG 13 & SG & 03617 & $B=18.57$ \\
\hline 2440836.46011 & 1970-09-06 & $22: 59$ & 30 & $\mathrm{I}-\mathrm{N}$ sen & RG 5 & SG & 03618 & $I=14.13$ \\
\hline 2440882.44129 & $1970-10-22$ & $22: 31$ & 30 & $103 \mathrm{a}-\mathrm{O}$ & GG 13 & SG & 03757 & $B=17.64$ \\
\hline 2440882.46560 & $1970-10-22$ & 23:06 & 30 & $\mathrm{I}-\mathrm{N}$ sen & RG 5 & SG & 03758 & $I=14.35$ \\
\hline 2440885.41835 & $1970-10-25$ & $21: 58$ & 30 & $103 \mathrm{a}-\mathrm{O}$ & GG 13 & SG & 03781 & $B>17.73$ \\
\hline 2440888.48568 & $1970-10-28$ & $23: 35$ & 30 & $\mathrm{I}-\mathrm{N}$ sen & RG 5 & SG & 03826 & $I=15.23$ \\
\hline 2440888.51207 & $1970-10-28$ & $24: 13$ & 30 & $103 \mathrm{a}-\mathrm{O}$ & GG 13 & SG & 03827 & $B=17.56$ \\
\hline 2440895.43001 & $1970-11-04$ & $22: 15$ & 40 & 103 a-E & RG 1 & SG & 03861 & $R=14.92$ \\
\hline 2440895.45779 & 1970-11-04 & $22: 55$ & 30 & $103 \mathrm{a}-\mathrm{O}$ & GG 13 & SG & 03862 & $B=17.56$ \\
\hline 2440918.23491 & $1970-11-27$ & $17: 35$ & 30 & $\mathrm{I}-\mathrm{N}$ sen & RG 5 & SG & 03951 & $I=15.15$ \\
\hline 2440918.25922 & $1970-11-27$ & $18: 10$ & 30 & $103 \mathrm{a}-\mathrm{O}$ & GG 13 & SG & 03952 & $B=18.57$ \\
\hline 2440924.31731 & $1970-12-03$ & $19: 34$ & 31 & $\mathrm{I}-\mathrm{N}$ sen & RG 5 & SG & 04019 & $I=14.86$ \\
\hline 2440924.34231 & $1970-12-03$ & $20: 10$ & 30 & $103 \mathrm{a}-\mathrm{O}$ & GG 13 & SG & 04020 & $B=18.32$ \\
\hline 2440941.25820 & $1970-12-20$ & $18: 10$ & 40 & II a-O & GG 13 & SG & 04084 & $B=18.32$ \\
\hline 2440941.28597 & $1970-12-20$ & $18: 50$ & 30 & $\mathrm{I}-\mathrm{N}$ sen & RG 5 & SG & 04085 & $I=14.86$ \\
\hline 2440970.23026 & $1971-01-18$ & $17: 32$ & 30 & $\mathrm{I}-\mathrm{N}$ sen & RG 5 & SG & 04149 & $I=14.98$ \\
\hline 2441179.47520 & $1971-08-15$ & $23: 22$ & 30 & $103 \mathrm{a}-\mathrm{O}$ & GG 13 & SG & 04574 & $B>17.73$ \\
\hline 2441179.50367 & $1971-08-15$ & $24: 03$ & 40 & 103 a-E & RG 1 & SG & 04575 & $R=15.66$ \\
\hline 2441179.53284 & $1971-08-15$ & $24: 45$ & 30 & I-N sen & RG 5 & SG & 04576 & $I=15.20$ \\
\hline 2441215.47854 & $1971-09-20$ & $23: 25$ & 30 & $0 \mathrm{a}-\mathrm{O}$ & GG 13 & SG & 04660 & $B=18.32$ \\
\hline 2441215.50910 & $1971-09-20$ & $24: 09$ & 40 & $\mathrm{I}-\mathrm{N}$ sen & RG 5 & SG & 04661 & $I=15.20$ \\
\hline 2441245.40311 & $1971-10-20$ & $21: 36$ & 20 & $103 \mathrm{a}-\mathrm{O}$ & GG 13 & SG & 04796 & $B=17.90$ \\
\hline 2441245.42325 & $1971-10-20$ & $22: 05$ & 30 & $\mathrm{I}-\mathrm{N}$ sen & RG 5 & SG & 04797 & $I=14.86$ \\
\hline 2441272.39362 & $1971-11-16$ & $21: 23$ & 30 & $103 \mathrm{a}-\mathrm{O}$ & GG 13 & SG & 04934 & $B=18.49$ \\
\hline 2441272.42556 & $1971-11-16$ & $22: 09$ & 40 & $\mathrm{I}-\mathrm{N}$ sen & RG 5 & SG & 04935 & $I=15.19$ \\
\hline 2441291.23044 & $1971-12-05$ & $17: 29$ & 25 & $103 \mathrm{a}-\mathrm{O}$ & GG 13 & SG & 04998 & $B=18.57$ \\
\hline 2441292.21373 & $1971-12-06$ & $17: 05$ & 35 & $\mathrm{I}-\mathrm{N}$ sen & RG 5 & SG & 05004 & $I=15.11$ \\
\hline 2441292.23803 & $1971-12-06$ & $17: 40$ & 25 & $103 \mathrm{a}-\mathrm{O}$ & GG 13 & SG & 05005 & $B=18.49$ \\
\hline 2441297.24753 & $1971-12-11$ & $17: 54$ & 40 & $\mathrm{I}-\mathrm{N}$ sen & RG 5 & SG & 05050 & $I=14.98$ \\
\hline 2441297.27600 & $1971-12-11$ & $18: 35$ & 30 & $\mathrm{Ia}-\mathrm{O}$ & GG 13 & SG & 05051 & $B=18.57$ \\
\hline 2441300.20851 & $1971-12-14$ & $16: 58$ & 40 & $\mathrm{I}-\mathrm{N}$ sen & RG 5 & SG & 05078 & $I=14.98$ \\
\hline 2441300.23628 & $1971-12-14$ & $17: 38$ & 30 & $\mathrm{Ia}-\mathrm{O}$ & GG 13 & SG & 05079 & $B>17.73$ \\
\hline 2441570.44841 & 1972-09-09 & $22: 42$ & 20 & $103 \mathrm{a}-\mathrm{O}$ & GG 13 & SG & 05598 & $\mathrm{~B}=18.57$ \\
\hline 2441570.47411 & 1972-09-09 & $23: 19$ & 30 & $\mathrm{I}-\mathrm{N}$ sen & RG 5 & SG & 05599 & $\mathrm{I}=14.98$ \\
\hline 2441606.40035 & $1972-10-15$ & $21: 32$ & 30 & I-N sen & RG 5 & SG & 05682 & $\mathrm{I}=15.28$ \\
\hline 2441606.42049 & $1972-10-15$ & $22: 01$ & 20 & $103 \mathrm{a}-\mathrm{O}$ & GG 13 & SG & 05683 & $B>17.73$ \\
\hline 2441622.39813 & $1972-10-31$ & $21: 29$ & 20 & $103 \mathrm{a}-\mathrm{O}$ & GG 13 & SG & 05724 & $B=18.57$ \\
\hline 2441622.42174 & $1972-10-31$ & $22: 03$ & 30 & $\mathrm{I}-\mathrm{N}$ sen & RG 5 & SG & 05725 & $I=15.03$ \\
\hline 2441626.34181 & $1972-11-04$ & $20: 08$ & 20 & $103 \mathrm{a}-\mathrm{O}$ & GG 13 & SG & 05776 & $B=17.54$ \\
\hline 2441626.36750 & $1972-11-04$ & $20: 45$ & 30 & $\mathrm{I}-\mathrm{N}$ sen & RG 5 & SG & 05777 & $I=15.12$ \\
\hline 2441649.22587 & $1972-11-27$ & $17: 22$ & 30 & $\mathrm{I}-\mathrm{N}$ sen & RG 5 & SG & 05908 & $I=14.92$ \\
\hline 2441649.24601 & $1972-11-27$ & $17: 51$ & 20 & $103 \mathrm{a}-\mathrm{O}$ & GG 13 & SG & 05909 & $B=18.57$ \\
\hline 2441659.28309 & $1972-12-07$ & $18: 45$ & 20 & 103 a-D & GG 14 & SG & 05972 & $V=16.01$ \\
\hline 2441659.30254 & $1972-12-07$ & $19: 13$ & 30 & $\mathrm{I}-\mathrm{N}$ sen & RG 5 & SG & 05973 & $I=14.92$ \\
\hline 2441665.45712 & $1972-12-13$ & $22: 56$ & 30 & $\mathrm{I}-\mathrm{N}$ sen & RG 5 & SG & 06004 & $I=14.92$ \\
\hline 2441665.47725 & $1972-12-13$ & $23: 25$ & 30 & 103 a-D & GG 14 & SG & 06005 & $V=15.85$ \\
\hline 2441675.21358 & $1972-12-23$ & $17: 06$ & 30 & $\mathrm{I}-\mathrm{N}$ sen & RG 5 & SG & 06018 & $I=14.98$ \\
\hline 2441675.23372 & $1972-12-23$ & $17: 35$ & 20 & 103 a-D & GG 14 & SG & 06019 & $V=16.24$ \\
\hline 2441958.54820 & $1973-10-02$ & $25: 05$ & 30 & I-N sen & RG 5 & SG & 06671 & $I>15.04$ \\
\hline 2441958.57111 & $1973-10-02$ & $25: 38$ & 20 & $103 \mathrm{a}-\mathrm{O}$ & GG 13 & SG & 06672 & $B=18.57$ \\
\hline 2441976.42464 & $1973-10-20$ & $22: 07$ & 30 & 103 a-D & GG 14 & SG & 06733 & $V=16.43$ \\
\hline 2441976.46491 & $1973-10-20$ & $23: 05$ & 20 & $103 \mathrm{a}-\mathrm{O}$ & GG 13 & SG & 06734 & $B>16.57$ \\
\hline 2441980.47391 & $1973-10-24$ & $23: 18$ & 30 & $\mathrm{I}-\mathrm{N}$ sen & RG 5 & SG & 06767 & $I=14.80$ \\
\hline 2441980.49683 & $1973-10-24$ & $23: 51$ & 20 & $103 \mathrm{a}-\mathrm{O}$ & GG 13 & SG & 06768 & $B>17.73$ \\
\hline
\end{tabular}


R. Jurdana-Šepić et al.: Towards a better classification of unclear eruptive variables

Table A.3. continued.

\begin{tabular}{|c|c|c|c|c|c|c|c|c|}
\hline HJD & date & UT & expt & emulsion & filter & tel & plate & mag \\
\hline 2441983.38291 & $1973-10-27$ & $21: 07$ & 20 & $103 \mathrm{a}-\mathrm{O}$ & GG 13 & SG & 06802 & $B=18.57$ \\
\hline 2441983.40166 & $1973-10-27$ & $21: 34$ & 20 & $103 \mathrm{a}-\mathrm{D}$ & GG 14 & $\mathrm{SG}$ & 06803 & $V=16.09$ \\
\hline 2441987.46410 & 1973-10-31 & 23:04 & 20 & $103 \mathrm{a}-\mathrm{O}$ & GG 13 & $\mathrm{SG}$ & 06850 & $B>18.57$ \\
\hline 2441987.48563 & $1973-10-31$ & $23: 35$ & 30 & I-N sen & RG 5 & SG & 06851 & $I>15.86$ \\
\hline 2442002.31516 & $1973-11-15$ & $19: 30$ & 20 & 103 a-D & GG 14 & SG & 06879 & $V=16.41$ \\
\hline 2442017.39450 & $1973-11-30$ & $21: 25$ & 30 & $103 \mathrm{a}-\mathrm{D}$ & GG 14 & SG & 06899 & $V=15.95$ \\
\hline 2442017.42228 & 1973-11-30 & $22: 05$ & 30 & $\mathrm{I}-\mathrm{N}$ sen & RG 5 & $\mathrm{SG}$ & 06900 & $I>15.04$ \\
\hline 2442029.24260 & $1973-12-12$ & $17: 47$ & 20 & 103 a-D & GG 14 & $\mathrm{SG}$ & 06923 & $V=16.67$ \\
\hline 2442029.26760 & $1973-12-12$ & $18: 23$ & 30 & I-N sen & RG 5 & $\mathrm{SG}$ & 06924 & $I>13.99$ \\
\hline 2442034.30694 & $1973-12-17$ & $19: 20$ & 30 & $\mathrm{I}-\mathrm{N}$ sen & RG 5 & SG & 06947 & $I=15.41$ \\
\hline 2442034.32777 & $1973-12-17$ & $19: 50$ & 20 & 103 a-D & GG 14 & SG & 06948 & $V=16.51$ \\
\hline 2442689.36765 & 1975-10-03 & $20: 45$ & 30 & $103 \mathrm{a}-\mathrm{O}$ & GG 13 & $\mathrm{SG}$ & 08102 & $B=17.90$ \\
\hline 2442689.38917 & $1975-10-03$ & $21: 16$ & 30 & I-N sen & RG 5 & SG & 08103 & $I=14.39$ \\
\hline 2442712.38848 & $1975-10-26$ & $21: 15$ & 30 & $103 \mathrm{a}-\mathrm{O}$ & GG 13 & SG & 08151 & $B=18.57$ \\
\hline 2442712.41626 & $1975-10-26$ & $21: 55$ & 30 & $\mathrm{I}-\mathrm{N}$ sen & RG 5 & SG & 08152 & $I=14.92$ \\
\hline 2442746.31470 & $1975-11-29$ & $19: 30$ & 20 & $103 \mathrm{a}-\mathrm{O}$ & GG 13 & $\mathrm{SG}$ & 08206 & $B=18.57$ \\
\hline 2442746.33693 & $1975-11-29$ & $20: 02$ & 30 & I-N sen & RG 5 & $\mathrm{SG}$ & 08207 & $I=14.92$ \\
\hline 2443039.46808 & $1976-09-17$ & $23: 10$ & 20 & $103 \mathrm{a}-\mathrm{O}$ & GG 13 & SG & 08684 & $B=18.07$ \\
\hline 2443039.48961 & 1976-09-17 & $23: 41$ & 30 & $\mathrm{I}-\mathrm{N}$ sen & RG 5 & SG & 08685 & $I=15.56$ \\
\hline 2443074.31698 & $1976-10-22$ & $19: 32$ & 30 & I-N sen & RG 5 & SG & 08722 & $I=14.86$ \\
\hline 2443074.34129 & $1976-10-22$ & $20: 07$ & 30 & $103 \mathrm{a}-\mathrm{O}$ & GG 13 & SG & 08723 & $B=18.49$ \\
\hline 2443127.39735 & $1976-12-14$ & $21: 30$ & 30 & $103 \mathrm{a}-\mathrm{O}$ & GG 13 & SG & 08797 & $B>17.73$ \\
\hline 2443127.42165 & $1976-12-14$ & $22: 05$ & 30 & I-N sen & RG 5 & SG & 08798 & $I=14.80$ \\
\hline 2443392.47259 & $1977-09-05$ & $23: 17$ & 30 & $\mathrm{I}-\mathrm{N}$ sen & RG 5 & SG & 09166 & $I=15.12$ \\
\hline 2443392.49481 & 1977-09-05 & $23: 49$ & 20 & 103 a-O & GG 13 & SG & 09167 & $B>17.73$ \\
\hline 2443398.46027 & 1977-09-11 & $22: 59$ & 30 & I-N sen & RG 5 & $\mathrm{SG}$ & 09199 & $I=14.92$ \\
\hline 2443398.48527 & 1977-09-11 & $23: 35$ & 20 & $103 \mathrm{a}-\mathrm{O}$ & GG 13 & SG & 09200 & $B>17.73$ \\
\hline 2443405.43198 & 1977-09-18 & $22: 18$ & 20 & $103 \mathrm{a}-\mathrm{O}$ & GG 13 & SG & 09213 & $B=17.54$ \\
\hline 2443405.46254 & 1977-09-18 & $23: 02$ & 30 & $\mathrm{I}-\mathrm{N}$ sen & RG 5 & $\mathrm{SG}$ & 09214 & $I=15.38$ \\
\hline 2443420.40376 & 1977-10-03 & $21: 37$ & 30 & $\mathrm{I}-\mathrm{N}$ sen & RG 5 & $\mathrm{SG}$ & 09224 & $I=14.92$ \\
\hline 2443452.27862 & 1977-11-04 & $18: 37$ & 30 & $\mathrm{I}-\mathrm{N}$ sen & RG 5 & SG & 09273 & $I=14.26$ \\
\hline 2443464.35194 & $1977-11-16$ & $20: 23$ & 30 & $\mathrm{I}-\mathrm{N}$ sen & RG 5 & $\mathrm{SG}$ & 09324 & $I=14.92$ \\
\hline 2443480.35415 & $1977-12-02$ & $20: 27$ & 30 & $103 \mathrm{a}-\mathrm{O}$ & GG 13 & SG & 09347 & $B>16.88$ \\
\hline 2443480.43262 & $1977-12-02$ & $22: 20$ & 30 & $\mathrm{I}-\mathrm{N}$ sen & RG 5 & $\mathrm{SG}$ & 09350 & $I=15.29$ \\
\hline 2443492.30361 & $1977-12-14$ & $19: 15$ & 30 & $\mathrm{I}-\mathrm{N}$ sen & RG 5 & SG & 09372 & $I>15.04$ \\
\hline 2443492.38139 & $1977-12-14$ & $21: 07$ & 30 & $103 \mathrm{a}-\mathrm{O}$ & GG 13 & SG & 09375 & $B>17.73$ \\
\hline 2443789.40935 & $1978-10-07$ & $21: 45$ & 30 & 103 a-D & GG 14 & SG & 09652 & $V=16.38$ \\
\hline 2443789.43366 & $1978-10-07$ & $22: 20$ & 30 & $\mathrm{I}-\mathrm{N}$ sen & RG 5 & SG & 09653 & $I=15.80$ \\
\hline 2444116.38069 & 1979-08-30 & $21: 05$ & 30 & $\mathrm{I}-\mathrm{N}$ sen & RG 5 & $\mathrm{SG}$ & 10162 & $I=15.19$ \\
\hline 2444116.49528 & 1979-08-30 & $23: 50$ & 20 & $103 \mathrm{a}-\mathrm{O}$ & GG 13 & $\mathrm{SG}$ & 10163 & $B>17.73$ \\
\hline 2444143.44325 & $1979-09-26$ & $22: 34$ & 30 & I-N sen & RG 5 & $\mathrm{SG}$ & 10186 & $I=14.98$ \\
\hline 2444143.47172 & $1979-09-26$ & $23: 15$ & 20 & $103 \mathrm{a}-\mathrm{O}$ & GG 13 & SG & 10187 & $B>17.73$ \\
\hline 2444172.45030 & $1979-10-25$ & $22: 44$ & 20 & $103 \mathrm{a}-\mathrm{O}$ & GG 13 & $\mathrm{SG}$ & 10249 & $B>17.73$ \\
\hline 2444172.47182 & $1979-10-25$ & $23: 15$ & 30 & $\mathrm{I}-\mathrm{N}$ sen & RG 5 & SG & 10250 & $I=14.98$ \\
\hline 2444552.47852 & $1980-11-08$ & $23: 25$ & 20 & 103 a-D & GG 14 & SG & 10668 & $V=15.62$ \\
\hline 2444557.42771 & 1980-11-13 & $22: 12$ & 20 & $103 \mathrm{a}-\mathrm{O}$ & GG 13 & SG & 10672 & $B>16.75$ \\
\hline 2444557.45479 & $1980-11-13$ & $22: 51$ & 30 & $\mathrm{I}-\mathrm{N}$ sen & RG 5 & $\mathrm{SG}$ & 10673 & $I>15.04$ \\
\hline 2444854.40387 & 1981-09-06 & $21: 38$ & 30 & $\mathrm{I}-\mathrm{N}$ sen & RG 5 & $\mathrm{SG}$ & 11099 & $I=15.19$ \\
\hline 2444911.34879 & 1981-11-02 & $20: 18$ & 30 & $\mathrm{I}-\mathrm{N}$ sen & RG 5 & SG & 11152 & $I=15.26$ \\
\hline 2444911.37726 & 1981-11-02 & $20: 59$ & 30 & $103 \mathrm{a}-\mathrm{O}$ & GG 13 & $\mathrm{SG}$ & 11153 & $B=17.85$ \\
\hline 2444933.41070 & $1981-11-24$ & $21: 48$ & 30 & $103 \mathrm{a}-\mathrm{O}$ & GG 13 & SG & 11241 & $B>17.73$ \\
\hline 2444933.44195 & $1981-11-24$ & $22: 33$ & 30 & $\mathrm{I}-\mathrm{N}$ sen & RG 5 & SG & 11242 & $I>15.04$ \\
\hline 2445264.32394 & 1982-10-21 & $19: 42$ & 25 & $\mathrm{I}-\mathrm{N}$ sen & RG 5 & SG & 11723 & $I=14.86$ \\
\hline 2445264.35310 & $1982-10-21$ & $20: 24$ & 30 & 103 a-D & GG 14 & SG & 11724 & $V=16.38$ \\
\hline 2445290.37764 & 1982-11-16 & $21: 00$ & 30 & $103 \mathrm{a}-\mathrm{O}$ & GG 13 & $\mathrm{SG}$ & 11740 & $B=17.98$ \\
\hline 2445591.41726 & 1983-09-13 & $21: 57$ & 30 & $\mathrm{I}-\mathrm{N}$ sen & RG 5 & SG & 12209 & $I=15.20$ \\
\hline 2445591.44296 & 1983-09-13 & $22: 34$ & 30 & 103 a-D & GG 14 & $\mathrm{SG}$ & 12210 & $V>16.63$ \\
\hline 2445611.46209 & 1983-10-03 & 23:01 & 30 & $103 \mathrm{a}-\mathrm{O}$ & GG 13 & $\mathrm{SG}$ & 12228 & $B=17.67$ \\
\hline 2449223.38669 & $1993-08-23$ & $21: 14$ & 30 & $103 \mathrm{a}-\mathrm{E}$ & RG 1 & SG & 15636 & $R>15.05$ \\
\hline
\end{tabular}


A\&A 614, A9 (2018)

Table A.4. Ranges of photometric variability for ASASSN-15qi.

\begin{tabular}{lccccc}
\hline \hline Source & Band & $N_{\text {obs }}$ & Median & $\begin{array}{c}\sigma \\
(\mathrm{mag})\end{array}$ & Peak \\
\hline \multirow{3}{*}{ ASASSN-15qi } & $B$ & 29 & 18.32 & 0.40 & 17.54 \\
& $V$ & 12 & 16.38 & 0.32 & 15.62 \\
& $R$ & 3 & 15.05 & 0.39 & 14.92 \\
& $I$ & 45 & 14.98 & 0.31 & 14.13 \\
\hline
\end{tabular}

Notes. For each band, we list the number of observations not considering the upper limits (Col. 3), the median, which basically indicates the magnitude in quiescence (Col. 4), the standard deviation data point distribution (Col. 5), and the magnitude corresponding to the peak brightness (Col. 6). 